\title{
CO-INFECÇÃO ENTRE CITOMEGALOVÍRUS CMV (HHV5) E HERPES VÍRUS HUMANO 6 (HHV6) EM RECEPTORES DE TRANSPLANTE DE RIM
}

\author{
CMV Co-infection (HHV5) and Human Herpes Virus 6 (HHV6) in renal transplant recipients.
}

\author{
Juliana Andréa Manfrinato', Janaína Luisa Leite², Sohemys Silvestre Bodine', Laura Sterian.Ward², Gentil Alves Filho', Marilda Mazzali'
}

\section{RESUMO}

Objetivo: Avaliar a ocorrência de co-infecção entre CMV-HHV6 em transplantados renais. Métodos: Critérios de inclusão: PCR para CMV negativo na ocasião do transplante, que se tornou positivo durante o acompanhamento. Dos 25 indivíduos que preenchiam os critérios de inclusão, foram selecionadas três amostras consecutivas de sangue, para pesquisa de HHV6 e CMV pela técnica de Nested PCR. Resultados: Por ocasião do transplante, $30 \%$ dos pacientes apresentavam HHV6-PCR positivo. Durante o acompanhamento, 11 pacientes adicionais apresentaram pelo menos um HHV6-PCR+, totalizando uma incidência de $80 \%$ de viremia para HHV6 nesta série. Como alguns autores sugerem que tanto HHV6 quanto HHV7 podem agravar o quadro clínico de infecção pelo CMV, a análise dos prontuários médicos mostrou que nove pacientes apresentavam viremia assintomática por CMV. Nos 16 casos restantes, foi identificada leucopenia isolada ( $\mathrm{n}=8$ ) ou infecção sintomática pelo CMV, com necessidade de tratamento com ganciclovir $(\mathrm{n}=8)$. Não houve diferença na incidência de viremia por HHV6 nos grupos com CMV assintomático ou leucopenia isolada. Entretanto, no grupo com infecção sintomática por CMV tratada, a prevalência de viremia por HHV6 foi de 100\%. A função renal dos diferentes grupos era comparável por ocasião da viremia. No entanto, após um ano de acompanhamento, houve uma tendência para níveis mais elevados de creatinina no grupo HHV6 positivo, que se tornou significativa após cinco anos $(2,5$ vs $1,2 \mathrm{mg} / \mathrm{dl}, \mathrm{p}<0,05)$. Conclusão: A co-infecção por CMV-HHV6 foi associada à apresentação clínica mais grave do CMV, com necessidade de terapia antiviral. A piora da função renal no grupo HHV6+ pode ser consequência de reduções temporárias de imunossupressão frente ao quadro infeccioso mais intenso, favorecendo a ocorrência de episódios de rejeição subclínica.

Descritores: Ctomegalovírus; Herpesviridae; Transplante Renal

Instituições:
1 Disciplina de Nefrologia - DCM; FCM Unicamp, Campinas, São Paulo, Brasil
2 Laboratório de Genética Molecular do Cancer (GEMOCA) - FCM Unicamp, Campinas/SP

Correspondência:

Marilda Mazzali, MD

Disciplina de Nefrologia DCM-FCM UNICAMP

Rua Tessália Vieira de Camargo, 126 - Cidade Universitária Zeferino Vaz - Barão Geraldo, CEP 13083-970, Campinas/SP, Brasil

Fone / Fax: (19) 3521-7959

Email: marildamazzali@gmail.com

\section{INTRODUÇÃO}

Herpes vírus humanos (HHV) são patógenos frequentes em seres humanos, persistindo no hospedeiro após a infecção primária. Infecções virais latentes são comuns em receptores de transplante de órgãos, podendo ser reativadas pelo uso de medicamentos imunossupressores. Um risco adicional para infecções virais e oportunistas nessa população é a contaminação através do órgão de doador soro positivo com desenvolvimento da infecção primária ou reativação de infecção latente. ${ }^{1}$

O Herpes vírus Humano 6 (HHV6) é um beta vírus herpes com patogênese ainda obscura, que atua como um patógeno oportunista em pacientes transplantados. ${ }^{2}$ A infecção primária pelo HHV6 ocorre na infância, sob forma de roseola infantum e, cerca de $90 \%$ das crianças menores de dois anos são soropositivas para HHV6. ${ }^{3}$ Após a infecção primária, o HHV6 permanece em estado latente em macrófagos, monócitos e em diferentes tecidos como pele, pulmão e cérebro. ${ }^{4}$ A reativação do HHV6 pode ocorrer após o transplante. No entanto, devido à alta prevalência de sorologia positiva para HHV6, da associação com outros herpes vírus e da 
ausência de grandes estudos populacionais, seu significado clínico permanece controverso. ${ }^{4}$ A apresentação clínica da infecção pelo HHV6 varia desde a forma assintomática até febre, supressão da medula óssea, encefalite, hepatite e pneumonia, além de facilitar a ocorrência de episódios de rejeição aguda, especialmente em pacientes transplantados renais pediátricos. ${ }^{5,6}$

A associação entre o herpes vírus tipo 6 (HHV6) e citomegalovírus (CMV-HHV5) foi descrita em diferentes séries. ${ }^{7-10}$ Estudos relatam que a co-infecção por HHV6 ou HHV7 aumenta o risco de infecções relacionadas ao CMV sintomático ${ }^{10-12}$ ou de episódios de rejeição [6]. Da mesma forma, CMV também pode causar reativação do HHV6, embora essas associações permaneçam controversas. ${ }^{13-15}$

O objetivo do presente estudo foi avaliar a incidência e o impacto clínico da infecção por HHV6 em receptores de transplante renal com viremia pelo CMV detectada pela Nested-PCR.

\section{MATERIAL E MÉTODOS Sujeitos da pesquisa}

O estudo foi aprovado pelo Comitê de Ética da Faculdade de Medicina da Universidade Estadual de Campinas (FCM / UNICAMP) e foi obtido consentimento informado de todos os sujeitos da pesquisa. A partir de um estudo prévio ${ }^{16}$ de 48 pacientes transplantados renais, foram selecionados 25 que preenchiam os critérios de inclusão:

(a) pelo menos três amostras de DNA adequado para análise,

(b) primeira amostra de DNA negativa para CMV

(c) DNA segunda amostra positiva para CMV / PCR

(d) terceiraamostra de DNAobtidapelomenos 30 dias após a segunda. Os prontuários médicos daqueles pacientes foram avaliados de forma retrospectiva para coleta de informações sobre os sintomas clínicos da doença CMV: leucograma, creatinina sérica e evolução clínica do paciente e do enxerto.

\section{Processamento das amostras de sangue:}

As amostras de sangue foram obtidas de todos os pacientes e células do sangue periférico (PBMC), foram isoladas por centrifugação, lavadas e armazenadas a $-20^{\circ} \mathrm{C}$ até o uso. O DNA foi extraído pela técnica de fenol-clorofórmio e etanol precipitado e os extratos foram armazenados em freezer a $-20^{\circ}$.C até o uso. A concentração de DNA foi determinada por espectrofotômetro 260/280nm.

A fim de amplificar as sequências do HHV-6, dois conjuntos de primers Nested foram utilizados. O primeiro conjunto amplificou a sequência correspondente ao gene da proteína principal do capsidium, consistindo de um par de primers externos, EX1 [5'GCG TCA TTT GTG TGT AGT ACG TCG G-3] e EX2 ['5'-TGG CCG CAT TCG GGA TAC TAC AGA GG-3' ] com sequência de $520 \mathrm{pb}$, e um par interno de primers, IN3 [5'-GCT ACG TAT TTG CTG CAG AAC G-3 '] IN4 [5'-ATC CGA AAC TGT CTG ACT GGC A-3 ' $](258 \mathrm{pb}) .{ }^{17} \mathrm{O}$ segundo conjunto de primers amplificou a região que codifica a proteína do tegumento, consistindo de um par de primers externos, A1 [-GAT CCG GTG ACG TCC TAC AAA CAC-3 '] e C1[/ 5'-CGG TGT CAC ACA GCA TGA ACT CTC-3 '] (834pb), e um par interno A2[5'-GGA GAA AAG TCT TGT ATG TAT GTC-3 '] e C2[5'-GGA CTC CTC ATA GAT CTG CTC ATA-3 '] com 658pb. Todas as reações de PCR foram realizadas em termocicladora programável (Perkin Elmer), com um volume final de $25 \mathrm{ml}$, incluindo $200 \mathrm{ng}$ de DNA, $50 \mathrm{mM}$ $\mathrm{KCl}, 20 \mathrm{mM}$ Tris-HCl, $15 \mathrm{mM} \mathrm{MgCl2,} \mathrm{0,2} \mathrm{mM} \mathrm{de} \mathrm{cada} \mathrm{dNTP,} \mathrm{e}$ $3 \mathrm{U}$ de Taq DNA polimerase (Life Technologies Gibco BRL). A temperatura de anelamento foi de $56^{\circ} \mathrm{C}$. Após 35 ciclos, $2 \mathrm{~m} 1$ do produto da PCR foi utilizado como modelo para uma segunda rodada de amplificação com IN3/IN4, nas mesmas condições de reação. A reação com o conjunto de primers $\mathrm{A} 1 / \mathrm{C} 1$ utilizou $25 \mathrm{ml}$ de cada primer externo, com 35 ciclos à temperatura de anelamento de $56^{\circ} \mathrm{C}$. Após a primeira amplificação, $2 \mathrm{ml}$ do produto da PCR foram amplificados, utilizando os primers internos $\mathrm{A} 2 / \mathrm{C} 2$ com 35 ciclos à temperatura de anelamento de 56 ${ }^{\circ} \mathrm{C}$, seguido por extensão à $72^{\circ} \mathrm{C}$ por $1,5 \mathrm{~min}$. Os produtos da amplificação foram visualizados em gél de agarose $2 \%$ contendo brometo de etídio, para avaliar a presença ou ausência de bandas em 258 bp (capsídeo protéico-6) e 658 pb (HHV HHV-6 proteína de tegumento).

Para as sequências HCMV foi utilizado conjunto de primers externos MIE4 [5'-AGC CCA GGC CTC TAG AT CCA AGC C-3'] e MIE 5 [5'-CAG CAC CAT CCT CCT CTT CCT CTG G-3'], com sequência de 435 pb e um par de primers interno, IE1 [5'-GTG CCA CCC CCA GTG GCT CC-3 '] e IE2 [5'-GCT CCC CCT CCT GAG CAC CC -3 '] com sequência alvo de $159 \mathrm{pb}[19,20]$. As reações foram realizadas em termocicladora programável (Perkin-Elmer), com volume final de $25 \mathrm{ml}$, incluindo $200 \mathrm{ng}$ de DNA, $50 \mathrm{~mm} \mathrm{KCl,} 20 \mathrm{mM}$ Tris-HCl, $15 \mathrm{mM}$ $\mathrm{MgCl} 2,0,2 \mathrm{mM}$ de cada dNTP, e $3 \mathrm{U}$ de Taq DNA polimerase (Life Technologies Gibco BRL). Os primers externos MIE4/ MIE5 foram utilizados em concentração final de $25 \mathrm{ml}$, com temperatura de anelamento de $55^{\circ} \mathrm{C}$. Após 30 ciclos, $2 \mathrm{ml}$ do produto da PCR foram utilizadas como modelo para uma segunda amplificação com os primers internos IE1/IE2, nas mesmas condições de experimento. Os produtos da amplificação foram visualizados em gél de agarose $2 \%$ contendo brometo de etídio para pesquisar bandas de DNA em $159 \mathrm{pb} .{ }^{18,19}$

Para evitar a possível contaminação de misturas de PCR, todas as reações foram calibradas com uma mistura de PCR sem DNA e água como controles negativos. O controle positivo do processo foi realizado com PCR para o gene da beta-globina. ${ }^{20}$

\section{ANÁLISE ESTATÍSTICA}

A análise estatística foi realizada através do programa Statview versão 2.0. Foram utilizados testes de qui-quadrado (x2) ou teste exato de Fisher (f) para variáveis qualitativas e testes de Mann Whitney para variáveis numéricas entre grupos. Foi considerada significância estatística $\mathrm{p}<0,05$.

\section{RESULTADOS}

Foram avaliados 25 transplantados renais (15 homens, 10 mulheres), com idade média de $38 \pm 10$ anos. A maioria (76\%) recebeu rim de doador falecido. A sorologia para CMV (IgG) foi positiva no pré-transplante em 23 indivíduos (92\%), todos CMVIgM negativos. Durante o acompanhamento, todos os pacientes tornaram-se CMVIgG positivos e seis apresentaram sorologia CMVIgM + transitória, sugerindo reativação da infecção em quatro pacientes previamente positivos e primo infecçção nos dois casos soronegativos. Todos os pacientes incluidos neste estudo 
apresentavam PCR negativo para CMV na ocasião do transplante, com positividade do exame (PCR) em cerca de $44 \pm 30$ dias após o transplante.

A apresentação clínica nos casos de viremia por CMV foi assintomática em nove, leucopenia isolada e leucopenia transitória em oito e presença de doença clínica e/ou antigenemia positiva com necessidade de terapia antiviral em oito pacientes $(32 \%$ da amostra).

Com o intuito de avaliar o impacto da co-infecção por HHV6 com a gravidade da viremia por CMV, realizamos Nested PCR para HHV6 (regiões 1 e 2) nas mesmas amostras previamente analisadas para CMV.

Por ocasião do transplante, $30 \%(\mathrm{n}=9)$ dos pacientes apresentaram viremia para HHV6 (PCR+), apesar do CMVPCR negativo. Durante o acompanhamento, outros 11 pacientes apresentaram pelo menos uma amostra positiva para particulas virais do HHV6 em sangue periférico.

Quando os grupos foram avaliados de acordo com HHV6 PCR, não observamos diferença nos parâmetros demográficos nem na ocorrência de viremia assintomática ou leucopenia isolada entre os grupos positivo e negativo (Tabela 1). Entretanto, observamos que a doença por CMV sintomática necessitando de terapia antiviral ocorreu somente no grupo HHV6PCR+ $(40 \%$ versus zero, $\mathrm{p}<0,05$ ) (Tabela 2).

Tabela 1: Dados demográficos de pacientes transplantados renais de acordo com o HHVGPCR.

\begin{tabular}{lll}
\hline & HHV6 negativo & HHV6 positivo \\
\hline $\begin{array}{l}\text { Número de pacientes } \\
\text { Sexo }\end{array}$ & 20 \\
(masculino:feminino) & $04: 01$ & $11: 09^{*}$ \\
$\begin{array}{l}\text { Idade (anos) } \\
\begin{array}{l}\text { Doador (falecido: } \\
\text { vivo relacionado) }\end{array}\end{array}$ & $04: 01$ & $39 \pm 11$ \\
$\begin{array}{l}\text { CMVIgG positivo } \\
\text { pré Tx }\end{array}$ & 18 & $15: 05$ \\
$\begin{array}{l}\text { CMVIgG negativo } \\
\text { pré TX }\end{array}$ & 2 & 5 \\
\hline
\end{tabular}

${ }^{*} \mathrm{p}<0,05$ entre grupos.

Tabela 2: Apresentação clínica da infecção pelo CMV de acordo com o status de HHVGPCR.

\begin{tabular}{lll}
\hline & HHV6 negativo & HHV6 positivo \\
\hline Assintomático & 6 & 3 \\
Leucopenia isolada & 6 & 2 \\
$\begin{array}{l}\text { Sintomático com } \\
\text { tratamento }\end{array}$ & 8 & $00^{*}$ \\
\hline
\end{tabular}

${ }^{*} p<0.05$
Como estudos anteriores sugeriram que a infecção viral seria um fator de risco para ocorrência de episódios de rejeição aguda ou disfunção crônica do enxerto, analisamos a função renal dos pacientes durante o acompanhamento. A creatinina sérica foi semelhante entre os grupos no momento do diagnóstico da viremia por CMV, com tendência para níveis mais elevados de creatinina após um e três anos de acompanhamento no grupo HHV6 positivo. Após 60 meses do transplante, a função renal foi pior no grupo HHV6 positivo (creatinina sérica 2,5 $\pm 0,4$ versus $1,3 \pm 0,1$; HHV6 positivo versus negativo, $\mathrm{p}<0,05)$, apesar de não haver diferença na incidência de rejeição aguda comprovada por biópsia entre os grupos.

\section{DISCUSSÃO}

Considerando que o CMV (HHV5) é provavelmente a infecção viral mais importante após transplante de órgãos, vários relatos sugeriram que outros beta herpes vírus como HHV-6 e HHV7 também podem ser ativados, causando sintomas clínicos após a introdução dos agentes imunossupressores. 1,9,10,12,13,21 Assim, com o advento de imunossupressores mais potentes, a incidência de infecções virais oportunistas, especialmente herpéticas, tende a aumentar. ${ }^{2,15}$

A associação da infecção pelo HHV6 com sintomas clínicos após o transplante não foi bem estabelecida. Como o contato com herpes vírus durante a infância é frequente, a prevalência de sorologia positiva varia de 12 a $82 \%$. $^{2,22} \mathrm{Na}$ presente série, a prevalência de HHV6PCR + foi de $80 \%$, superior a dados observados previamente em uma população semelhante. ${ }^{23}$ Essa alta prevalência pode ser influenciada pela seleção dos pacientes, uma vez que apenas pacientes com CMV PCR positiva foram incluídos nessa análise.

A apresentação usual da infecção pelo HHV-6 é assintomática ou com febre e exantema, e sua relevância clínica ainda é controversa tanto em indivíduos normais quanto em pacientes imunodeprimidos. ${ }^{1,2,22}$ No entanto, após o uso de imunossupressores mais potentes, a reativação de infecções latentes pode ser associada a quadro clínico mais grave, com leucopenia, hepatite, pneumonite e encefalite..$^{2,3,5}$ Alguns estudos sugerem que a ativação de mais de um beta herpes vírus pode explicar a maior gravidade da doença por CMV. ${ }^{14}$ Na presente série, o grupo HHV6 negativo apresentou viremia assintomática. No entanto, no grupo HHV6 positivo, observamos maior incidência (40\%) de doença sintomática por CMV com antigenemia positiva e necessidade de terapia antiviral.

Outra complicação associada à infecção por herpes vírus seria maior risco de ocorrência de episódios de rejeição aguda, além de infecções bacterianas e fúngicas oportunistas. ${ }^{5.24}$ Estudos prévios sugerem que o HHV-6 possa ter um efeito citopático direto sobre células epiteliais tubulares, aumentando o risco de nefropatia crônica do enxerto. ${ }^{25}$ Todos esses fatores causam impacto negativo na sobrevida tanto do enxerto como do paciente. Na presente série, apesar da menor incidência de rejeição aguda comprovada por biópsia no grupo HHV6 positivo, observamos pior função renal nos enxertos ao longo do acompanhamento. Houve tendência de aumento da creatinina sérica após um ano de acompanhamento, com taxa de filtração glomerular significativamente menor no grupo HHV6 positivo depois de cinco anos. Essa disfunção do enxerto 
pode ser secundária a um ajuste nas doses dos medicamentos imunossupressores (especialmente retirada ou redução de micofenolato) frente a quadros de infecção viral mais grave, com risco aumentado de episódios de rejeição aguda subclínica. Por outro lado, a presença de HHV6 pode ser um marcador de estados de imunossupressão mais intensa, favorecendo a nefrotoxicidade de imunossupressores, especialmente dos inibidores da calcineurina. Outra possibilidade para a disfunção renal não investigada nesta série seria o efeito citopático direto do HHV6 em células epiteliais tubulares, como sugerido por Hoshino et al. ${ }^{25}$

\section{CONCLUSÃO}

Em resumo, na presente série, presença de HHV6PCR positivo foi associado a um quadro mais severo de infecção por CMV, com necessidade de tratamento antiviral e ajuste da imunossupressão, o que em longo prazo pode apresentar impacto negativo sobre a função renal.

\section{Agradecimentos}

Agradecemos a Elisabeth Santos Costa e Sandra Andrella pela coleta das amostras de sangue dos pacientes transplantados incluídos neste estudo.

\section{ABSTRACT}

Purpose: The aim of this study was to assess the occurrence of co-infection of CMV-HHV6 bearers among 25 kidney transplant patients. Methods: Inclusion criteria were CMVPCR negative at the time of transplantation, which became positive during follow-up. Three consecutive blood samples were selected from each patient and analyzed both for HHV6 PCR and nested PCR for CMV. Results: At transplantation, $30 \%$ of patients were positive for HHV6PCR. During follow up, 11 additional patients had at least one HHV6PCR positive, resulting in a total of $80 \%$ HHV6 viremia in this series. As some authors suggest that both HHV6 and HHV7 may aggravate the clinical picture of CMV infection, medical records of those patients were analyzed. Asymptomatic CMV viremia was found in 9 patients. Remaining 16 patients had isolated leukopenia $(\mathrm{n}=8)$ or symptomatic CMV treatment with ganciclovir $(\mathrm{n}=8)$. While HHV6 PCR positive or negative was comparable between patients with asymptomatic CMV viremia or associated with transient leukopenia in the group treated with CMV disease, the prevalence of HHV6 positive blood was $100 \%$. No differences in renal function were observed during the viremia. However, after a year monitoring, there was a trend for higher levels of creatinine in the group positive for HHV6, which became significant after five years $(2.5 \mathrm{vs} .1 .2 \mathrm{mg} / \mathrm{dl}, \mathrm{p}<0.05)$. Conclusion, CMV/HHV6 co-infection is associated with more pronounced clinical symptoms and the need for antiviral treatment. The decline of renal function in group HHV6 positive can present a most serious consequence for CMV infection, a temporary immunosuppressive reduction or subclinical acute rejection episodes. Prospective studies are needed to answer these questions.

Keywords: Cytomegalovirus, herpesviridae, Kidney Transplantation

\section{REFERÊNCIAS:}

1. Smith SR, Butterly DW, Alexander BD, Grrenberg A. Viral infections after renal transplantation. Am J Kidney Dis. 2001;37(4):659-76.

2. Singh N, Carrigan DR. Human herpes virus 6 in transplantation. Ann Int Med. 1996;124(12):1065-71.

3. Leach CT, Sumaya CV, Brown NA. Human herpes virus 6: clinical implications of a recently discovered, ubiquitous agent. J Pediatr. 1992;121(2):173-81.

4. Gentile G. Post transplant hhv6 disease. Herpes. 2000;7(1):24-7.

5. Accot PD, Lee SH, Bitter Suermann H, Lawen JG, Crocker JF. Infections concomitant with pediatric renal allograft rejection. Transplantation. 1996;62:689-91.

6. Wade A, McDonald A, Accot PD, Lee S, Crocker JF. Human herpes virus 6 (HHV6) or Epstein Barr (EBV) infection and acute allograft rejection in pediatric kidney transplant recipients. Greater risk for immunologically naive recipients. Transplant Proc. 1998;30:2091-3.

7. Osman HK, Peiris JS, Taylor CE, Karlberg JP, Madeley CR. Correlation between the detection of viral dna by the polymerase chain reaction in peripheral blood leukocytes and serological responses to human herpesvirus 6, human herpesvirus 7, and cytomegalovirus in renal allograft recipients. J Med Virol. 1997;53(3):288-94.

8. Desjardin JA, Cho E, Supran S, Gibbons 1, Werner BG, Snydman DR. Association of human herpesvirus 6 reactivation with severe cytomegalovirus-associated disease in orthotopic liver transplant recipients. Clin Infect Dis. 2001;33(8):1358-62.

9. Desjardin JA, Gibbons 1, Cho E, Supran SE, Falagas ME, Werner BG, Snydman DR. Human herpesvirus 6 reactivation is associated with cytomegalovirus infection and syndromes in kidney transplant recipients at risk for primary cytomegalovirus infection. J Infect Dis. 1998;178(6):1783-6.

10. Ratnamohan VM, Chapman J, Howse H, Bovington K, Robertson P, Byth K, Allen R, Cunningham AL. Cytomegalovirus and human herpesvirus 6 both cause viral disease after renal transplantation. Transplantation. 1998;66(7):877-82.

11. Pacsa AS, Essa S, Voevodin A, et al. Correlation between genotypes, multiple infections with herpesviruses (HHV-6, 7) and development of disease in kidney recipients in Kuwait. Fems Immunol Med Microbiol. 2003;35(2):125-30.

12. Humar a, malkan g, moussa g, greig p, levy g, mazzulli t. Human herpesvirus-6 is associated with cytomegalovirus reactivation in liver transplant recipients. $\mathrm{J}$ infect dis. $2000 ; 181(4): 1450-3$.

13. Dockrell DH, Prada J, Jones MF, et al. Seroconversion to human herpesvirus 6 following liver transplantation is a marker of cytomegalovirus disease. J Infect Dis. 1997;176(5):1135-40.

14. Humar A, Kumar D, Raboud J, Caliendo AM, Moussa G, Levy G, Mazzulli T. Interactions between cytomegalovirus, human herpesvirus-6, and the recurrence of hepatitis C after liver transplantation. Am J Transplant. 2002 may;2(5):461-6.

15. Jha R, Narayen G, Sinha S, Kadeer K, Prasad KN. Symptomatic herpes virus infections in postrenal transplant. Transplant Proc. 2003;35(1):284-5.

16. Costa SCB, Miranda SRP, Alves G, Rossi CL, Figueiredo LTM, Costa FF. Detection of cytomegalovirus infection by PCR in renal transplant patients. Brazilian J Med Biol Res. 1999;32:953-9. 
17. Aubin JT, Collandre H, Candotti D et al. Several groups among human herpesvirus 6 strains can be distinguished by southern blotting and polymerase chain reaction. J Clin Microbiol. 1991;29:367-72.

18. Demmler GJ, Buffone GJ, Shimbor CM, Ray RA. Detection of cytomegalovirus in urine from newborns by using polymerase chain reaction DNA amplification. J Infect Dis. 1988;158:1177-84

19. Shibata D, Martin WJ, Appleman MD et al. Detection of cytomegalovirus DNA in peripheral blood of patients infected with human immunodefficiency virus. J Infec Dis. 1988;158:1185-92.

20. Saiki RK, Scharf SJ, Faloona F. Enzymatic amplification of b globin genomic sequence and restrition site analysis for diagnosis of sickle cell anemia. Science. 1985,230:1350-4.
21. Okuno T, Higashi K, Shiraki K et al. Human herpes virus 6 infection in renal transplantation. Transplantation. 1990;49:519-22.

22. Dockrell DH, Mendez JG, Jones MF et al. Human herpes virus 6 seronegativity before transplantation predicts the occurrence of fungal infection in liver transplant recipients. Transplantation. 1999;67:399-403

23. Leite JL, Manfrinato J, Alves-Filho G, Ward LS, Mazzali M. Infecção pelo herpes virus humano tipo 6 (HHV6) em um grupo de receptores de transplante renal. Jornal Brasileiro de Transplantes. 2006;(9):566-9.

24. Rubin RH. Infectious disease complications of renal transplantation. Kidney Int. 1993 jul;44(1):221-36.

25. Hoshino K, Nishi T, Adachi $\mathrm{H}$ et al. Human herpesvirus-6 infection in renal allografts: retrospective immunohistochemical study injapanese recipients. Transpl Int. 1995;8:169-73. 